\title{
La résistance aux antituberculeux au Canada : 2006 à 2016
}

\author{
V Gallant ${ }^{1}, J^{2}$ Vachon ${ }^{1 *}$, W Siu ${ }^{1}$
}

\section{Résumé}

Contexte : Les souches de bacilles tuberculeux pharmacorésistantes menacent grandement de saper les efforts de prévention et de lutte contre la tuberculose. Le Système canadien de surveillance des laboratoires de tuberculose (SCSLT) a été créé en 1998 pour surveiller les tendances et les profils émergents de la résistance aux antituberculeux au Canada.

Objectif : Présenter un survol descriptif des données sur la résistance aux antituberculeux recueillies par l'entremise du SCSLT pour les années 2006 à 2016 au Canada, en mettant l'accent sur 2016.

Méthodologie : Le SCSLT est un système de surveillance axé sur les isolats qui vise à recueillir des données sur la résistance aux antituberculeux dans tout le Canada. Chaque année, les données sont recueillies et analysées par l'Agence de la santé publique du Canada (ASPC), puis validées par le laboratoire qui les soumet.

Résultats : En 2016, les résultats des épreuves de sensibilité aux antituberculeux ont été rapportés pour 1452 isolats. La proportion de souches de tuberculose pharmacorésistantes est demeurée relativement stable, avec 108 (7,4\%) des isolats classés comme monorésistants, cinq $(0,3 \%)$ comme polyrésistants et $17(1,2 \%)$ comme souches multirésistantes à la tuberculose (TB-MR). En 2016, aucun isolat de tuberculose ultrarésistante (TB-UR) n'a été relevé. Les hommes représentaient $792(54,5 \%)$ de tous les isolats déclarés et 64 (49,2\%) des souches résistantes tandis que les femmes représentaient $11(64,7 \%)$ des souches de TB-MR. Entre 2006 et 2016, les personnes âgées de 15 à 44 ans représentaient 47,4 \% de tous les isolats déclarés, $54 \%$ des isolats présentant une résistance et $72,3 \%$ des souches TB-MR.

Conclusion : Les niveaux de résistance aux antituberculeux ont été relativement faibles et stables au cours des 11 dernières années et sont restés inférieurs à la moyenne mondiale depuis le début de la surveillance nationale. Cependant, l'inquiétude croissante suscitée dans le monde par la résistance aux antituberculeux et par l'émergence de souches de tuberculose ultrarésistante fait ressortir le rôle vital que continuera à jouer le Système canadien de surveillance des laboratoires de tuberculose dans la surveillance de la résistance aux antituberculeux au Canada.

\begin{abstract}
Affiliations
${ }^{1}$ Centre de la lutte contre les maladies transmissibles et les infections, Agence de la santé publique du Canada, Ottawa (Ontario)
\end{abstract}

*Correspondance : $t b$

surveillance@phac-aspc.gc.ca
Citation proposée : Gallant V, Vachon J, Siu W. La résistance aux antituberculeux au Canada : 2006 à 2016.

Relevé des maladies transmissibles au Canada. 2017;43(11):269-75. https://doi.org/10.14745/ccdr.v43i11a05f

\section{Introduction}

La tuberculose (TB), une maladie infectieuse transmissible par voie aérienne et causée par la bactérie Mycobacterium tuberculosis, demeure une cause majeure de morbidité et de mortalité dans de nombreuses régions du monde. En 2015, on estimait à 10,4 millions le nombre de nouveaux cas de tuberculose dans le monde et 1,8 million de personnes sont décédées de cette maladie guérissable (1). Bien que la majorité des cas de tuberculose soient causés par des souches sensibles aux meilleurs médicaments antituberculeux disponibles, la résistance aux médicaments suscite une préoccupation majeure pour le contrôle de la tuberculose. Alors que la durée moyenne du traitement d'une personne atteinte de tuberculose pleinement sensible varie entre six et neuf mois, le traitement de la tuberculose pharmacorésistante peut nécessiter de 12 à 18 mois (et peut-être plus longtemps) avec des médicaments plus coûteux, mais moins efficaces et potentiellement plus toxiques (2).
Les souches de bacilles tuberculeux pharmacorésistantes menacent grandement de saper les efforts de prévention et de lutte contre la tuberculose. Bien que la tuberculose pharmacorésistante ne soit pas un problème de santé publique majeur au Canada, elle représente tout de même un problème potentiel puisque les Canadiens voyagent fréquemment à l'étranger et de nombreuses personnes s'établissant au Canada proviennent de pays où l'incidence de la tuberculose, de même que la pharmacorésistance associée, sont élevées.

Le Système canadien de surveillance des laboratoires de tuberculose (SCSLT) a été créé en 1998. Il s'agit d'une collaboration entre les gouvernements fédéral, provinciaux et territoriaux et le Réseau technique canadien des laboratoires de tuberculose (RTCLT), un réseau pancanadien composé de directeurs techniques ou scientifiques de laboratoires de tuberculose provinciaux et territoriaux. Le système est géré 
par le Centre de la lutte contre les maladies transmissibles et les infections (CLMTI) de l'Agence de la santé publique du Canada (ASPC).

Le principal objectif du Système canadien de surveillance des laboratoires de tuberculose est de surveiller les tendances et les profils émergents de la résistance aux antituberculeux au Canada. Ce rapport de surveillance présente un aperçu descriptif des données pour les années 2006 à 2016, en mettant l'accent sur l'année 2016, sur la résistance aux antituberculeux majeurs et mineurs au Canada. Les résultats sont répartis par province ou territoire et, dans la mesure du possible, par sexe et par groupe d'âge. Le SCSLT saisit des données démographiques minimales (âge, sexe et province ou territoire seulement). Par conséquent, aucune observation directe n'est faite sur la pharmacorésistance liée à d'autres facteurs démographiques, y compris l'origine ethnique. En tant que principale source de données nationales sur la résistance aux antituberculeux au Canada, les données présentées dans le présent rapport ont pour but d'éclairer les mesures de santé publique, ainsi que l'élaboration et l'évaluation des politiques et des programmes.

Auparavant, ces données étaient publiées chaque année dans un rapport distinct intitulé La tuberculose : La résistance aux antituberculeux au Canada. Il s'agit de la première version du rapport à être publiée dans le Relevé des maladies transmissibles au Canada (RMTC). Les données présentées dans le présent rapport sont les plus récentes au moment de la publication et remplacent les données publiées précédemment. Des tableaux de données supplémentaires sont accessibles en ligne (3).

\section{Méthodologie}

Le SCSLT est un système de surveillance basé sur les isolats qui vise à recueillir des données sur la résistance aux antituberculeux dans tout le Canada. La résistance aux antituberculeux est déterminée au moyen d'épreuves de sensibilité sur des échantillons biologiques (isolats) prélevés chez des personnes atteintes de tuberculose à culture positive (2). Des renseignements détaillés sur les méthodes de collecte de données du SCSLT, la gestion des données et d'autres processus de laboratoire ont déjà été présentés (4). De même, une liste des antituberculeux majeurs et mineurs recommandés et les concentrations critiques recommandées pour le dépistage systématique sont présentées (4).

Tous les laboratoires participants ont testé la résistance aux quatre des antituberculeux majeurs (isoniazide, éthambutol, rifampicine et pyrazinamide), à l'exception du Laboratoire de microbiologie et de référence en santé publique de la Colombie-Britannique, qui n'a pas systématiquement testé la résistance au pyrazinamide. Si une résistance à I'un des trois autres médicaments majeurs est détectée, la Colombie-Britannique analyse alors l'isolat pour évaluer la résistance au pyrazinamide. Pour tous les laboratoires, les résultats des épreuves de sensibilité aux antituberculeux mineurs ont été soumis pour les isolats présentant une résistance à l'isoniazide et à la rifampicine en vue de déterminer la tuberculose ultrarésistante (TB-UR) des isolats. Le tableau 1 décrit les profils de résistance aux médicaments antituberculeux tels qu'ils sont définis dans les Normes canadiennes pour la lutte antituberculeuse (2).

Les résultats des épreuves de sensibilité aux médicaments (sensible/résistant/non effectuée) pour tous les isolats du complexe Mycobacterium tuberculosis (CMTB) mis en évidence
Tableau 1 : Définitions des profils de résistance aux antituberculeux (2)

\begin{tabular}{|l|l|}
\hline \multicolumn{1}{|c|}{ Profil de résistance } & \multicolumn{1}{c|}{ Définition } \\
\hline Monorésistance & $\begin{array}{l}\text { Résistance à un seul antituberculeux } \\
\text { de première intention (isoniazide, } \\
\text { rifampicine, éthambutol ou } \\
\text { pyrazinamide). }\end{array}$ \\
\hline Polyrésistance & $\begin{array}{l}\text { Résistance à plus d'un antituberculeux } \\
\text { de première intention, à l'exception de } \\
\text { la combinaison isoniazide et rifampicine. }\end{array}$ \\
\hline $\begin{array}{l}\text { Tuberculose multirésistante } \\
\text { (TB-MR) }\end{array}$ & $\begin{array}{l}\text { Résistance à l'isoniazide ET à la } \\
\text { rifampicine avec ou sans résistance } \\
\text { à d'autres antituberculeux. }\end{array}$ \\
\hline $\begin{array}{l}\text { Tuberculose ultrarésistante } \\
\text { (TB-UR) }\end{array}$ & $\begin{array}{l}\text { Résistance à l'isoniazide ET à la } \\
\text { rifampicine ET à toute fluoroquinolone } \\
\text { ET à au moins un des trois } \\
\text { antituberculeux injectables de deuxième } \\
\text { intention (amikacine, capréomycine ou } \\
\text { kanamycine). }\end{array}$ \\
\hline
\end{tabular}

par culture, plus précisément $M$. tuberculosis, M. africanum, M. canetti, M. caprae, M. microti, M. pinnipedii ou M. bovis, ont été soumis volontairement à l'ASPC par les laboratoires provinciaux de lutte antituberculeuse pour inclusion dans le SCSLT. Les données ont été soumises à I'ASPC, soit par I'entremise d'un formulaire de déclaration normalisé rempli manuellement (formulaire intitulé Rapport sur la sensibilité des souches du complexe M. tuberculosis aux antimicrobiens) ou par voie électronique (5).

Des procédures normalisées d'enregistrement des données ont été appliquées à toutes les données afin de créer un ensemble de données nationales. Toutes les données brutes (formulaires papier et électroniques) ont été conservées conformément à la directive de I'ASPC sur la collecte, l'utilisation et la diffusion de l'information sur la santé publique.

Aucune procédure statistique n'a été utilisée pour les analyses comparatives, et aucune technique statistique n'a été appliquée pour tenir compte des données manquantes. Les données des tableaux contenant de petites cellules $(n=<5)$ n'ont pas été supprimées puisqu'elles ne semblaient pas présenter de risque d'identification de cas individuels. Ces procédures étaient conformes à la directive sur la collecte, l'utilisation et la diffusion de l'information sur la santé publique. Les données présentées dans le présent rapport ont été extraites de la base de données du SCSLT en mars 2017 et ont été validées par les laboratoires déclarants. Les logiciels Microsoft Excel 2010 et SAS Enterprise Guide (SAS EG) v5.1 ont été utilisés pour le nettoyage et I'analyse des données.

\section{Résultats}

En 2016, les résultats des épreuves de sensibilité aux antituberculeux ont été présentés à I'ASPC pour 1463 isolats. De ce nombre, $11(0,8 \%)$ étaient identifiés comme $M$. bovis Bacillus Calmette-Guerin et ont été exclus des analyses ultérieures. Parmi les 1452 isolats restants, 843 (58,1\%) ont été déclarés comme faisant partie d'un complexe Mycobacterium tuberculosis dont l'espèce était connue (830 étaient identifiés comme $M$. tuberculosis, neuf comme $M$. africanum et quatre comme M. bovis), et 609 (41,9\%) ont été déclarés comme appartenant à 
un complexe Mycobacterium tuberculosis d'une espèce inconnue (données non illustrées) (tableau supplémentaire 1).

\section{Caractéristiques de la résistance aux antituberculeux}

En 2016, 1322 (91\%) des patients isolés soumis au test étaient sensibles aux quatre antituberculeux majeurs. La proportion d'isolats résistants à la tuberculose est demeurée faible, 108 $(7,4 \%)$ des isolats étant classés comme monorésistants, cinq $(0,3 \%)$ comme polyrésistants et $17(1,2 \%)$ comme souches multirésistantes à la tuberculose (TB-MR) (figure 1).

Figure 1 : Profils de résistance aux antituberculeux selon le pourcentage d'isolats testés au Canada, 2006 à 2016

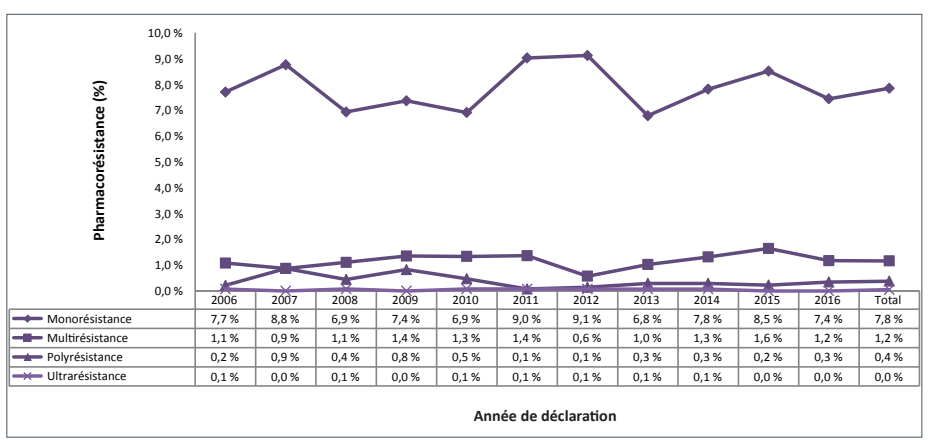

Pour la période de 2006 à 2016, des résultats d'épreuves de sensibilité ont été déclarés pour 14872 isolats (tableau supplémentaire 1). La monorésistance était la tendance la plus fréquente, représentant environ 1167 (7,8\%) de tous les isolats testés au cours de la période. Au total, $173(1,2 \%)$ des isolats ont été identifiés comme étant des isolats de tuberculose multirésistante (à l'exception de la tuberculose ultrarésistante) et sept $(<0,1 \%)$ des isolats ont été identifiés comme étant ultrarésistants. Bien qu'il y ait eu de légères fluctuations dans la proportion d'isolats présentant divers profils de résistance, les résultats demeurés constants au cours de la période de 11 ans allant de 2006 à 2016 (figure 2; tableau supplémentaire 2).

Figure 2 : Pourcentage $d$ 'isolats soumis à des épreuves de résistance à l'isoniazide, au pyrazinamide, à la rifampicine et à l'éthambutol, Canada, 2006 à 2016

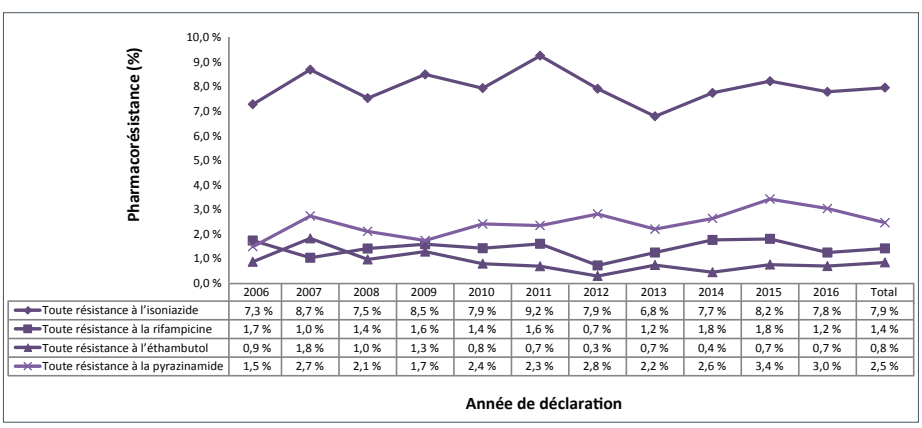

\section{Résistance aux antituberculeux majeurs}

En 2016, les résultats de sensibilité à l'éthambutol, à l'isoniazide et à la rifampicine étaient disponibles pour l'ensemble des 1452 isolats. On a rapporté des résultats d'épreuves de sensibilité au pyrazinamide pour 1254 (86,4 \%) isolats. Selon le pourcentage d'isolats testés, 113 (7,8 \%) étaient résistants à l'isoniazide, 38 (3,0 \%) étaient résistants au pyrazinamide, $18(1,2 \%)$ étaient résistants à la rifampicine et $10(0,7 \%)$ étaient résistants à l'éthambutol (tableau supplémentaire 2). Au total, $130(9,0 \%)$ des isolats étaient résistants à au moins un antituberculeux majeur. Entre 2006 et 2016, la proportion d'isolats déclarés présentant une résistance à au moins un des quatre antituberculeux mineurs était stable au fil du temps (figure 2). La résistance à l'isoniazide seul ou en association avec d'autres médicaments variait entre 7,5 et 9,2\% des isolats testés. La résistance à l'éthambutol, à la rifampicine et au pyrazinamide est demeurée inférieure à 3,5\% (figure 2).

\section{Monorésistance}

En 2016, 130 (9,0 \%) du nombre total d'isolats de la tuberculose ont été déclarés résistants à au moins un des quatre antituberculeux majeurs. De ce nombre, 108 (83,1\%) étaient monorésistants. Parmi les isolats monorésistants, 91 (84,3\%) étaient résistants à l'isoniazide, $16(14,8 \%)$ au pyrazinamide et un $(0,9 \%)$ à la rifampicine. Aucun isolat n'a été trouvé monorésistant à l'éthambutol (tableau supplémentaire 3 - tableau 15; données non répertoriées dans les tableaux).

Entre 2006 et 2016, 1167 (7,8\%) isolats se sont avérés monorésistants à l'un des quatre antituberculeux majeurs. De ce nombre, la résistance à l'isoniazide était la plus souvent signalée avec $946(81,1 \%)$ des isolats (figure 3$)$. Au cours de cette période, $29(2,5 \%)$ des isolats monorésistants se sont avérés résistants à la rifampicine. En moyenne, un à trois isolats de rifampicine monorésistants ont été signalés chaque année entre 2006 et 2016 (figure 3; tableau supplémentaire 3 - tableau 15; données non répertoriées dans les tableaux).

Figure 3 : Pourcentage d'isolats monorésistants résistants à l'isoniazide, au pyrazinamide, à la rifampicine ou à l'éthambutol, Canada, 2006 à 2016



\section{Tuberculose polyrésistante, tuberculose} multirésistante et tuberculose ultrarésistante

\section{aux antituberculeux}

En 2016, cinq $(0,2 \%)$ des isolats étaient résistants à au moins deux antituberculeux majeurs (à l'exception de la combinaison de l'isoniazide et de la rifampicine) et ont donc été classés comme étant polyrésistants. Deux isolats étaient résistants à I'isoniazide et à l'éthambutol, tandis que trois autres étaient résistants à l'isoniazide et au pyrazinamide.

Entre 2006 et 2016, $54(0,4 \%)$ des isolats ont été déclarés comme étant polyrésistants. De ce nombre, 23 (42,6\%) étaient résistants à l'isoniazide et à l'éthambutol, 24 (44,4\%) étaient résistants à l'isoniazide et au pyrazinamide et l'un d'entre eux 
était résistant à l'éthambutol et au pyrazinamide. Les six autres isolats $(11,1 \%)$ étaient résistants à l'isoniazide, à l'éthambutol et au pyrazinamide (tableau 3 supplémentaire - tableau 15; données non répertoriées dans les tableaux).

En 2016, 17 (1,2\%) des isolats testés étaient résistants à I'isoniazide et à la rifampicine. De ce nombre, six (35,3\%) étaient résistants seulement à l'isoniazide et à la rifampicine, quatre $(23,5 \%)$ étaient résistants à l'isoniazide, à la rifampicine et à l'éthambutol et trois $(17,6 \%)$ étaient résistants à l'isoniazide, à la rifampicine et au pyrazinamide. Enfin, quatre $(23,5 \%)$ des isolats étaient résistants à chacun des quatre antituberculeux majeurs (figure 4; tableau supplémentaire 17).

Figure 4 : Nombre et pourcentage d'isolats résistants à I'isoniazide et à la rifampicine avec ou sans résistance à l'éthambutol ou au pyrazinamide, Canada, 2006 à 2016

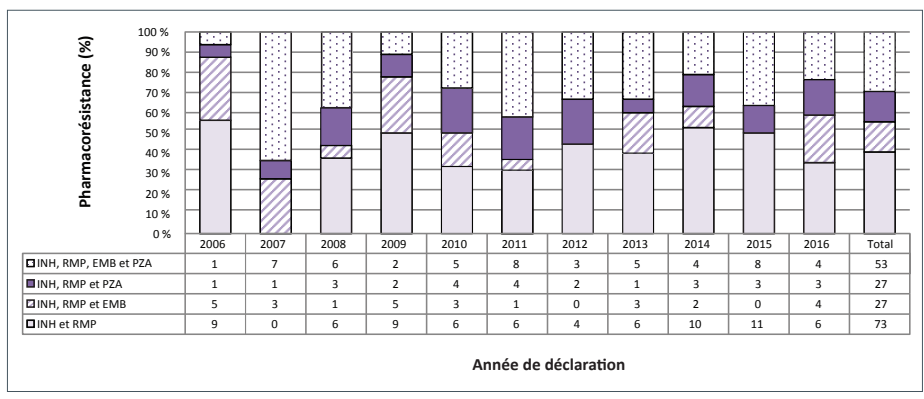

Abréviations : $\mathrm{EMB}$, éthambutol; INH, isoniazide; $\mathrm{PZA}$, pyrazinamide; RMP, rifampicine

Pour la période de 2006 à 2016, 180 (1,2\%) des isolats testés étaient résistants à la fois à l'isoniazide et à la rifampicine. Au cours de cette période, 73 (40,6\%) étaient résistants uniquement à l'isoniazide et à la rifampicine, 27 (15,0\%) étaient également résistants à l'éthambutol et 27 (15,0\%) étaient résistants au pyrazinamide. Les 53 autres isolats $(29,4 \%)$ étaient résistants aux quatre antituberculeux majeurs (figure 4 ; tableau supplémentaire 3 - tableau 15; données non répertoriées dans les tableaux).

Pour déterminer la tuberculose ultrarésistante, tous les isolats qui se sont révélés résistants à la fois à l'isoniazide et à la rifampicine ont été soumis ensuite à des épreuves de sensibilité à certains antituberculeux mineurs. En 2016, sur les 17 isolats identifiés comme étant résistants à la fois à l'isoniazide et à la rifampicine, un isolat était résistant à au moins un des agents injectables (amikacine, capréomycine ou kanamycine), mais sensible aux fluoroquinolones, et deux isolats étaient résistants à au moins une fluoroquinolone, mais sensibles à l'ensemble des agents injectables. Les 14 autres isolats étaient tous sensibles à la fois aux antituberculeux injectables et aux fluoroquinolones. Comme aucun des 17 isolats résistants à l'isoniazide et à la rifampicine n'était résistant à la fois à un agent injectable et à une fluoroquinolone, aucun isolat n'a été classé comme étant un TB-UR. L'année 2016 a été la deuxième année consécutive où aucun isolat de tuberculose ultrarésistante n'a été signalé.

Entre 2006 et 2016, sur les 180 isolats résistants à la fois à I'isoniazide et à la rifampicine, 141 (78,3 \%) se sont révélés sensibles aux agents injectables et aux fluoroquinolones (figure 5). En outre, 18 (10,0\%) étaient résistants aux agents injectables, mais sensibles aux fluoroquinolones et 14 (7,8 \%) étaient résistants aux fluoroquinolones, mais sensibles aux agents injectables. Enfin, sept $(3,9 \%)$ des isolats se sont révélés résistants aux fluoroquinolones et aux agents injectables, ce qui en fait des souches de tuberculose ultrarésistante (figure 5).
Figure 5 : Nombre et pourcentage d'isolats résistants à l'isoniazide et à la rifampicine avec ou sans résistance aux fluoroquinolones ou aux agents injectables, Canada, 2006 à 2016

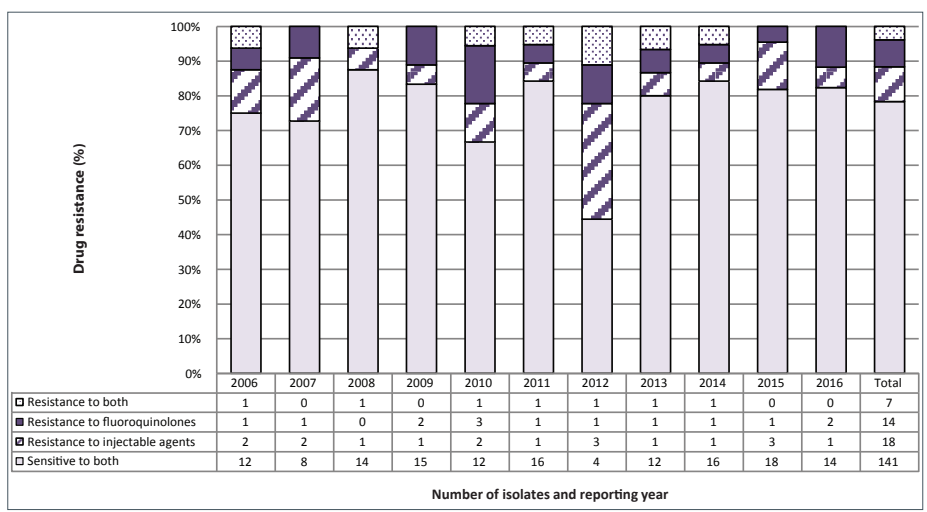

\section{Répartition géographique}

En 2016, 1314 (90,4 \%) isolats provenaient de cinq provinces : I'Ontario, le Québec, la Colombie-Britannique, I'Alberta et le Manitoba (figure 6; tableau supplémentaire 16). La Saskatchewan représentait moins de $5 \%$ des isolats déclarés, tandis que les territoires du Nord (Territoires du Nord-Ouest, Nunavut et Yukon) et les provinces de I'Atlantique (Nouveau-Brunswick, Terre-Neuve-et-Labrador, Nouvelle-Écosse et île-du-Prince-Édouard) représentaient moins de $5 \%$ des isolats déclarés. En 2016, tous les isolats provenant de Nunavut, des Territoires du Nord-Ouest, du Yukon, de Terre-Neuve-et-Labrador, de Nouveau-Brunswick, et de l'île-du-Prince-Édouard étaient sensibles à tous les antituberculeux majeurs testés. Parmi les 17 isolats de tuberculose multirésistante, sept $(41,2 \%)$ provenaient de l'Ontario, 4 (23,5\%) de l'Alberta et deux (11,8\%) de la Colombie-Britannique, du Québec et du Manitoba.

Figure 6 : Nombre d'isolats du complexe Mycobacterium tuberculosis déclarés, par province ou territoire d'origine et sous forme de pourcentage de tous les isolats déclarés au Canada, 2016

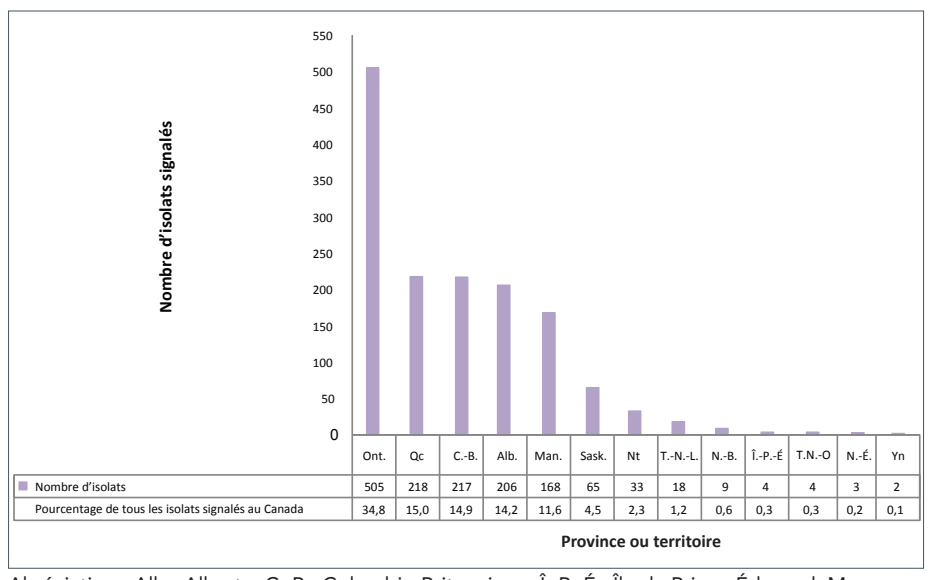

Abréviation : Alb., Alberta, C.-B., Colombie-Britannique, Î.-P.-É., Île du Prince-Édouard; Man., Manitoba, N.-B., Nouveau-Brunswick; N.-É., Nouvelle-Écosse, Nt., Nunavut; Ont., Ontario, Qc, Québec; Sask., Saskatchewan; T.-N.-L., Terre-Neuve-et-Labrador; T.N.-O., Territoires du NordOuest; Yn, Yukon

Pour la période de 2006 à 2016, les 173 isolats de tuberculose multirésistante provenaient de sept provinces : l'Alberta, la 
Colombie-Britannique, le Manitoba, le Nouveau-Brunswick, I'Ontario, le Québec et la Saskatchewan. L'Ontario représentait $97(56,1 \%)$ de tous les isolats de tuberculose multirésistante et a déclaré, en moyenne, neuf isolats de TB-MR par année (plage : 6 à 14) (tableau supplémentaire 11). L'Alberta représentait $24(13,9 \%)$ des isolats de tuberculose multirésistante déclarés, dont plus de 10 (41,6\%) ont été signalés entre 2014 et 2016 (tableau supplémentaire 3).

Entre 2006 et 2016, la Colombie-Britannique et le Québec ont en moyenne déclaré systématiquement moins de deux isolats de TB-MR par année. Le Manitoba a déclaré sept isolats de TB-MR au cours des onze dernières années, soit en moyenne moins d'un isolat par année. Le Nouveau-Brunswick et la Saskatchewan ont déclaré un et deux isolats de TB-MR respectivement, entre 2006 et 2016 (tableau supplémentaire 18).

Entre 2006 et 2016, sept isolats de TB-UR ont été signalés. Parmi les sept, cing provenaient de l'Ontario, un du Manitoba et un du Québec (tableau supplémentaire 18).

\section{Données démographiques}

En 2016, le sexe était connu en ce qui concerne 1450 (99,9\%) des 1452 personnes dont les isolats ont été prélevés (tableau supplémentaire 19). Bien que les hommes représentaient $732(54,5 \%)$ de tous les isolats déclarés et $64(49,2 \%)$ des isolats résistants, les femmes représentaient $11(64,7 \%)$ des isolats de tuberculose multirésistante.

Entre 2006 et 2016, les hommes représentaient 8265 (56,3\%) de tous les isolats déclarés, $726(53,1 \%)$ des isolats présentant une certaine résistance et $85(50 \%)$ des isolats de tuberculose multirésistante (données non présentées). Bien qu'il n'y ait eu que sept isolats de tuberculose ultrarésistante déclarés entre 2006 et 2016, cinq provenaient des femmes. La proportion de femmes dont la tuberculose a présenté une pharmacorésistance a légèrement augmenté au cours de la période de 11 ans (passant de $8,1 \%$ en 2006 à $10 \%$ en 2016) et était plus élevée que chez les hommes (8,1\%) en 2016 (figure 7).

Figure 7 : Pourcentage des isolats présentant une résistance chez tous les isolats testés, selon le sexe au Canada, 2006 à 2016

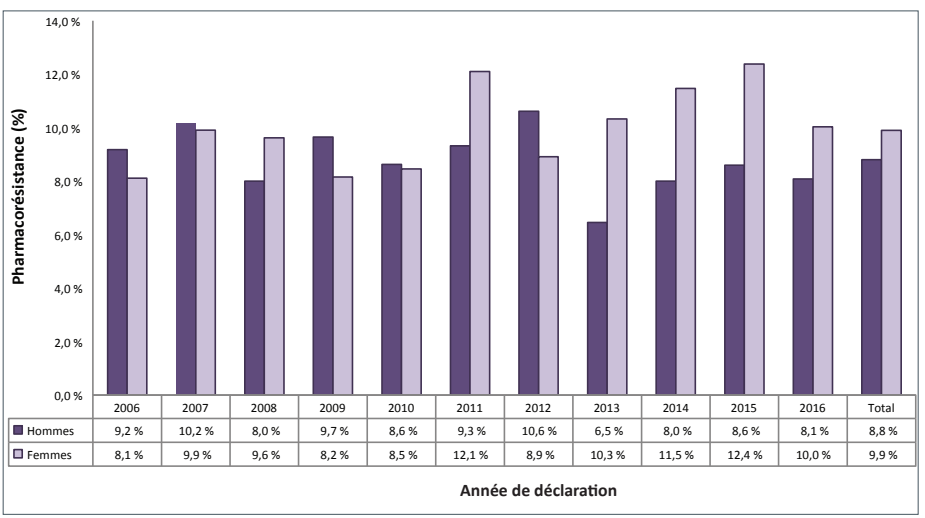

En 2016, l'âge ou la date de naissance ont été déclarés pour l'ensemble des personnes chez lesquelles les 1452 isolats ont été prélevés (tableau supplémentaire 19). Parmi les 130 isolats pharmacorésistants, 26 (20\%) des personnes présentant une certaine résistance ont été prélevés chez des personnes âgées de 25 à 34 ans et $25(19,2 \%)$ ont été observés chez les personnes âgées de 15 à 24 ans (tableau supplémentaire 19). Cinq isolats présentant une résistance quelconque provenaient de sujets âgés de moins de 15 ans. En 2016, 16 (94,1\%) des 17 cas de tuberculose multirésistante concernaient des personnes âgées de 15 à 64 ans et un seul cas a été relevé chez une personne âgée de 5 à 14 ans (tableau supplémentaire 19).

Entre 2006 et 2016, les personnes âgées de 15 à 44 ans représentaient $7045(47,4 \%)$ de tous les isolats déclarés, 757 $(54 \%)$ des isolats présentant une résistance et $125(72,3 \%)$ des isolats de TB-MR (figure 8).

Figure 8 : Proportion des isolats déclarés selon le profil de résistance et le groupe d'âge au Canada, 2006 à 2016

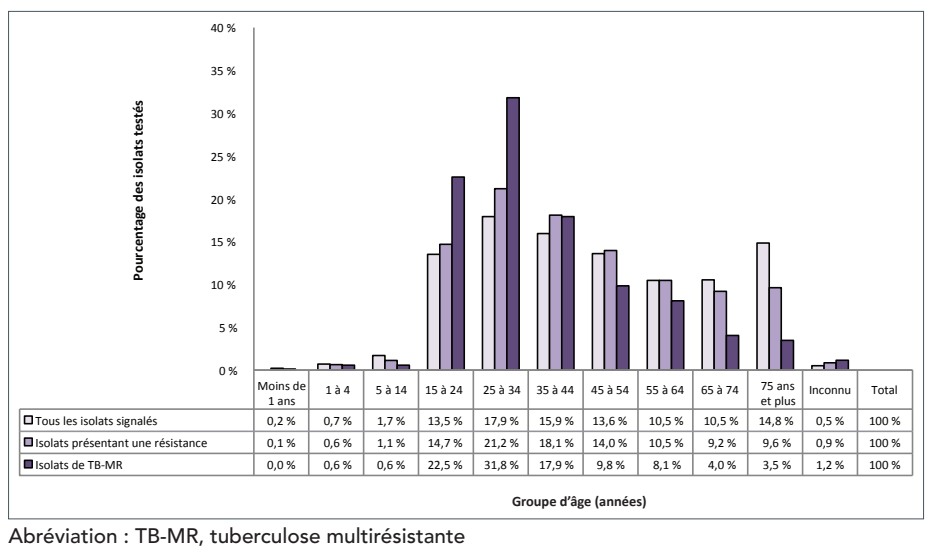

\section{Discussion}

Au Canada, entre 2006 et 2016, les tendances de la résistance aux antituberculeux sont demeurées relativement faibles et stables. En 2016, bien qu'il y ait eu une légère augmentation du nombre d'isolats déclarés par rapport aux années précédentes, cela ne s'est pas traduit par une augmentation de la pharmacorésistance. Parmi les résultats soumis en $2016,9 \%$ de tous les isolats testés étaient résistants à au moins un des quatre antituberculeux majeurs, la majorité $(83,1 \%)$ d'entre eux étaient résistants à un seul médicament. La tuberculose multirésistante n'a été relevée que dans 1,2\% des isolats testés tandis que la tuberculose ultrarésistante n'a pas été relevée parmi les résultats déclarés en 2016.

La monorésistance à l'isoniazide a été la tendance la plus souvent observée au Canada. La rifampicine, ainsi que l'isoniazide, est I'un des médicaments majeurs les plus efficaces; heureusement, la monorésistance à la rifampicine demeure faible, mais une surveillance continue demeurera importante pour déceler tout changement dans les profils de résistance à ce médicament.

En 2016, les données ont démontré une certaine variation régionale, y compris une légère augmentation des niveaux de tuberculose multirésistante en Alberta. De plus, la proportion de femmes présentant une résistance semblait augmenter légèrement au fil du temps et était plus élevée que celle des hommes présentant une résistance.

Dans de nombreuses régions du monde, la pharmacorésistance constitue une difficulté majeure à surmonter pour la prévention et le contrôle de la tuberculose. L'Europe de l'Est et l'Asie centrale continuent d'afficher la proportion la plus élevée de cas de tuberculose multirésistante dans le monde (1). Les souches de tuberculose qui sont résistantes à la fois à l'isoniazide et à la 
rifampicine rendent les efforts de traitement et de prévention considérablement plus difficiles, car la disponibilité des antituberculeux efficaces devient limitée.

Comme il est indiqué dans La tuberculose au Canada - Résumé de 2015, les personnes nées à l'étranger ont continué à représenter la majorité des cas déclarés, soit $71 \%$ des cas de tuberculose active diagnostiqués au Canada (6) et il est probable que ces personnes aient contracté l'infection à l'extérieur du Canada. Par conséquent, bien que les données sur le pays d'origine ne soient pas recueillies dans le SCSLT, les profils de résistance aux antituberculeux au Canada sont probablement influencés par des profils de pharmacorésistance ailleurs dans le monde.

Les données publiées par l'Organisation mondiale de la Santé indiquent qu'en 2015, à l'échelle mondiale, environ 3,9\% (intervalle de confiance à $95 \%: 2,7 \%$ à 5,1\%) des nouveaux cas de tuberculose et $21 \%$ (intervalle de confiance à $95 \%: 15 \%$ à $28 \%$ ) des cas de tuberculose déjà traités étaient des cas de TB-MR (1). Bien que les données recueillies au moyen du SCSLT n'établissent pas de distinction entre les isolats des nouveaux cas de tuberculose et ceux des cas déjà traités, il est rassurant de constater que seulement 1,2\% des isolats testés en 2016 étaient des cas de TB-MR (ce qui est nettement inférieur aux estimations mondiales). En outre, l'identification de seulement sept cas de tuberculose ultrarésistante pendant la période de 2006 à 2016 indique que la tuberculose ultrarésistante reste une maladie rare au Canada.

Les divers rapports ont des limites dont il faut tenir compte. Malgré les efforts déployés pour s'assurer que les déclarations multiples pour une même personne au cours d'une année donnée aient été supprimées, il est possible, compte tenu des renseignements d'identification minimaux disponibles pour chaque isolat (âge et sexe), que des déclarations multiples pour une même personne aient été incluses dans la base de données. Ce biais est probablement minime compte tenu du processus de validation auprès des fournisseurs de données provinciaux et territoriaux.

Les données démographiques et cliniques recueillies par l'entremise du SCSLT étaient limitées et aucune donnée n'a été recueillie sur l'origine ethnique, l'état diagnostique/clinique ou les résultats du traitement de la personne auprès de laquelle l'échantillon a été prélevé. Une plus grande quantité de données démographiques et de données cliniques permettrait de réaliser une évaluation épidémiologique plus approfondie des profils de pharmacorésistance au Canada. D'après les données recueillies à partir de ce système de surveillance (1), il n'a pas été possible de distinguer une pharmacorésistance primaire d'une résistance acquise ni de faire la différence entre les profils de résistance des nouveaux cas et ceux des cas traités de nouveau. Toutefois, les rapports de surveillance La tuberculose au Canada - Résumé de 2015 (6) et La tuberculose au Canada 2012 (7) donnent un aperçu du nombre total de cas de tuberculose actifs déclarés et des taux d'incidence correspondants au Canada selon certaines caractéristiques démographiques et cliniques, présentent des données basées sur des cas (et non sur des isolats) sur la pharmacorésistance primaire et acquise au Canada, lesquelles n'étaient pas présentées ici. Ensemble, ces rapports donnent un aperçu complet des données de surveillance des cas de tuberculose et de pharmacorésistance à l'échelle nationale.

Habituellement, seuls les isolats de tuberculose multirésistante ou d'autres profils de tuberculose ultrarésistante font l'objet d'épreuves de sensibilité à certains antituberculeux mineurs. Bien que le Clinical and Laboratory Standards Institute (CLSI) recommande d'évaluer la résistance aux antituberculeux mineurs des isolats monorésistants à l'isoniazide, ainsi que des autres isolats polyrésistants, mais non multirésistants (8), cette recommandation n'est pas toujours respectée au Canada. Les isolats autres que ceux de la tuberculose multirésistante peuvent être résistants à une fluoroquinolone, car cette famille $d^{\prime}$ antibiotiques est largement utilisée dans le traitement des infections respiratoires. Dans une certaine mesure, notre compréhension de l'émergence de la résistance aux antituberculeux mineurs au Canada en est donc limitée.

L'Agence de la santé publique du Canada continue de travailler avec ses partenaires provinciaux et territoriaux pour atteindre l'objectif de l'élimination de la tuberculose au Canada. L'inquiétude croissante suscitée dans le monde par la résistance aux antituberculeux et par l'émergence de souches de tuberculose ultrarésistante fait ressortir le rôle vital que continue à jouer le Système canadien de surveillance des laboratoires de tuberculose dans la surveillance de la résistance aux antituberculeux au Canada.

\section{Déclaration des auteurs}

V.G. - Conceptualisation, méthodologie, logiciels, validation, analyse officielle et rédaction - Ébauche originale

J.V. - Conceptualisation, rédaction - Examen et révision

W.S. - Rédaction - Examen, révision et supervision

\section{Conflit d'intérêt}

Aucun.

\section{Remerciements}

La Division de surveillance de la santé et de l'épidémiologie du Centre de lutte contre les maladies transmissibles et les infections de l'Agence de la santé publique du Canada tient à remercier les membres du Réseau technique canadien de laboratoires de tuberculose et leurs équipes, ainsi que les collègues du Laboratoire national de microbiologie, pour leur contribution et leur participation au Système canadien de surveillance des laboratoires de tuberculose.

\section{Financement}

Ce travail a été appuyé par l'Agence de la santé publique du Canada dans le cadre de son mandat de base.

\section{Références}

1. Organisation mondiale de la santé. Rapport sur la lutte contre la tuberculose dansl le monde. Genève: OMS Press; 2017. http://www.who.int/tb/publications/global_report/fr/

2. Menzies R, Wong $\mathrm{T}$ (eds). Normes Canadiennes pour la lutte antituberculeuse $7^{\mathrm{e}}$ édition. Société canadienne de thoracologie. L'Association pulmonaire du Canada, I'Agence santé publique du Canada; 2014. https://www.canada.ca/ content/dam/phac-aspc/migration/phac-aspc/tbpc-latb/ 
pubs/tb-canada-7/assets/pdf/tb-standards-tb-normes-preffra.pdf

3. V Gallant, J Vachon, W Siu. Tuberculosis drug resistance in Canada: 2006-2016 Supplementary data [Internet].

Can Commun Dis Rep. 2017;43(11). https://www. canada.ca/fr/public-health/services/reports-publications/ canada-communicable-disease-report-ccdr/monthlyissue/2017-43/ccdr-volume-43-11-2-novembre-2017/ resistance-antituberculeux-canada-donneessupplementaires-2006-2016.html

4. Agence de la santé publique du Canada. La tuberculose : La résistance aux antituberculeux au Canada 2015, Ottawa (Canada): Ministre des Travaux publics et des Services gouvernmentaux Canada; 2017. https://www.canada.ca/fr/ sante-publique/services/publications/maladies-et-affections/ tuberculose-resistance-aux-antituberculeux-canada-2015. html

5. Agence de la santé publique du Canada. Rapport sur la sensibilité des souches du complexe M. tuberculosis aux antimicrobiens. http://www.phac-aspc.gc.ca/tbpc-latb/pdf/ dsrf-fra.pdf

6. Gallant V, Duvvuri V, McGuire M. La tuberculose au Canada - Résumé 2015. Relevé des maladies transmissibles au Canada. 2017;43(3):77-82. https://www.canada.ca/content/ dam/phac-aspc/migration/phac-aspc/publicat/ccdrrmtc/17vol43/dr-rm43-3-4/assets/pdf/17vol43_3_4-ar-04-fra. pdf

7. Agence de la santé publique du Canada. La tuberculose au Canada 2012. Ottawa (Canada). http://www.phac-aspc. gc.ca/tbpc-latb/pubs/tbcan12/assets/pdf/tbcan12-fra.pdf

8. Woods GL, Brown-Elliott BA, Conville PS, Desmond EP, Lin G, Pfyffer GE, Ridderhof JC, Siddigi SH, Wallace RJ, Warren NG, Witebsky FG. Susceptibility testing of mycobacteria, nocardiae, and other aerobic actinomycetes; approved standard-Second Edition. Wayne, PA: Clinical and Laboratory Standards Institute; 2011:31(5). 